\title{
The OmniTread serpentine robot with pneumatic joint actuation ${ }^{*}$
}

\author{
Grzegorz GRANOSIK ${ }^{2}$ and Johann BORENSTEIN ${ }^{1}$ \\ ${ }^{1}$ Dept. of Mechanical Engineering \\ The University of Michigan \\ Ann Arbor, MI, USA \\ ${ }^{2}$ Institute of Automatic Control \\ Technical University of Łódź \\ Łódź, POLAND \\ johannb@umich.edu \\ granosik@p.lodz.pl
}

\begin{abstract}
This paper describes the design of a pneumatic joint actuation system for the OmniTread serpentine robot, developed at the University of Michigan. Serpentine robots are a new class of mobile robots with one unique characteristic: they have a very large ratio of length to diameter. To implement this unique shape, serpentine robots usually have multiple segments connected by joints. Some serpentine robots provide legged, wheeled, or tracked propulsion, and, in addition, actuation for the joints. In this paper we present a detailed analysis of pneumatic Integrated Joint Actuators (IJA) invented and built especially for serpentine robots. The IJA combines advantages of pneumatic bellows-like actuators with our proportional position and stiffness (PPS) control algorithm. Controllable stiffness is of crucial importance in serpentine robots, which require stiff joints to cross gaps and compliant joints to conform to rough terrain for effective propulsion. The paper also includes results of tests performed at the Southwest Research Institute.
\end{abstract}

Index Terms - Serpentine robot, pneumatic, joint, actuator, bellows, stiffness, compliance, control.

\section{INTRODUCTION}

Serpentine robots are slender, multi-segmented vehicles designed to provide greater mobility than conventional wheeled or tracked robots. We limit our definition of a "serpentine robot" to a mechanism that derives propulsion from wheels, legs, or tracks. Joints connecting the segments may be either powered or unpowered. We use the term "snake robot" for those robots that derive propulsion from undulations, that is, wave-like motions of the joints only, without wheels, legs, or tracks.

\section{A. Background}

The first practical realization of a serpentine robot, called KR-I, was introduced by Hirose and Morishima [1990] and the improved version KR-II was presented by Hirose et al. [1991]. This first serpentine robot was large and heavy, weighing in at $350 \mathrm{~kg}$. The robot comprises of multiple vertical cylindrical segments on powered wheels (tracks in KR-I) that give the mechanism a train-like appearance. Vertical joint actuators allow a segment to lift its neighbours up, in order to negotiation steps or span gaps. More recently, Klaassen and Paap [1999] at the GMD developed the Snake2 vehicle, which contains six active segments and a head. Each round segment has an array of 12 electrically driven wheels evenly spaced around its periphery. These wheels provide propulsion regardless of the vehicles orientation (i.e., its roll angle). Segments are interconnected by universal joints actuated by three additional electric motors through strings. Snake2 is equipped with six infrared distance sensors, three torque sensors, one tilt sensor, two angle sensors in every segment, and a video camera in the head segment. Snake2 was specifically designed for the inspection of sewage pipes.

Another serpentine robot designed for sewer inspection was developed by Scholl et al. [2000] at the Forschungszentrum Informatik (FZI) in Germany. Its segments use only two wheels but the actuated 3-DOF joints allow full control over each segment's spatial orientation. The sensor suite of this robot is similar to that of Snake2. The robot is able to negotiate tight $90^{\circ}$ angled pipes and climb over $55 \mathrm{~cm}$ high obstacles. One segment and its joint are about $20 \mathrm{~cm}$ long. The development of sewer inspection robots is continued in the joint project MAKROplus [Streich and Adria 2004].

While wheeled serpentine robots can work well in smoothwalled pipes, more rugged terrain requires tracked propulsion. To this effect Takayama and Hirose [2000] developed the Soruyu-I crawler, which consists of three segments. Each segment is driven by a pair of tracks, which, in turn, are all powered simultaneously by a single motor, located in the center segment. Torque is provided to the two distal segments through a rotary shaft and universal joints. Each distal segment is connected to the center segment by a special 2-DOF joint mechanism, which is actuated by two lead screws driven by two electric motors. The robot can move forward and backward, and it can change the orientation of the two distal segments in yaw and pitch symmetrically to the center segment. One interesting feature is the ability of this robot to adapt to irregular terrain because of the elasticity of its joints. This elasticity is provided by springs and cannot be actively controlled.

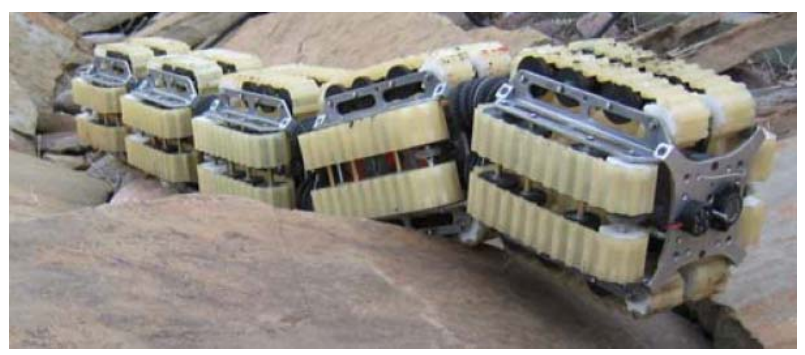

Figure 1: The OmniTread serpentine robot developed at University of Michigan. All segments are linked by Integrated Joint Actuators.

\footnotetext{
"This work was conducted at the University of Michigan’s Mobile Robotics Lab and funded by the U.S. Department of Energy under Award No. DE-FG0486NE3796 and by the Intelligence Technology Innovation Center (ITIC) through Southwest Research Institute under contract No. F009822.
} 
A serpentine robot that is strikingly similar to our OmniTread serpentine robot is MOIRA [Osuka and Kitajima, 2003] ${ }^{\dagger}$. MOIRA comprises four segments, and each segment has two longitudinal tracks on each of its four sides, for a total of eight tracks per segment. The 2-DOF joints between segments are actuated by pneumatic cylinders. We believe that the bellows-based joint actuators used in our OmniTread have a substantial advantage over a cylinder-based design, as the discussion of our approach in the next section will show.

A different concept using unpowered joints was introduced by Kimura and Hirose [2002] at the Tokyo Institute of Technology. That robot, called Genbu, is probably the only serpentine robot with unpowered joints. The stability of the robot and its high mobility on rough terrain are preserved by large-diameter wheels $(220 \mathrm{~mm})$. The control system employs position and torque feedback sensors for the passive but rigid joints. Springs are used to protect the electric motors from impact, although the stiffness of the springs cannot be controlled during operation.

The concept of joining several small robots into a train to overcome larger obstacles was used by researchers from Carnegie Mellon University in their Millibot Train [Brown et al. 2002]. This robot consists of seven electrically driven, very compact segments. The diameter of the track sprockets is larger than the height of each segment, which allows the robot to drive upside-down. Segments are connected by couplers for active connection and disconnection of segments, but the joints have only one DOF. Each joint is actuated by an electric motor with a high-ratio harmonic gear and slip clutch. It provides sufficient torque to lift up the three front segments. The robot has been demonstrated to climb up a regular staircase and even higher steps. However, with only one DOF in each joint the vehicle is kinematically limited.

\section{THE OMNITREAD DESIGN}

The OmniTread design offers two unique and fundamentally important advantages over all other serpentine robots described in the scientific literature to date. These features are: (1) maximal coverage of the robot's surface with propulsion elements and (2) joint actuation with pneumatic bellows.

\section{A. Complete coverage of robot with propulsion elements}

This patented design feature makes the serpentine robot indifferent to rolling over - a condition that is inevitable when the slender bodies of serpentine robots travel over rugged terrain. Another reason for the use of wide tracks on each side of each segment is our fundamental design doctrine that calls for covering the largest possible surface area of the robot with propulsion elements. The reason is that any contact between an environmental feature and a robot's inert (i.e., not propelling) surface impedes motion or entirely stops the robot (i.e., the robot gets “stuck"). In contrast, any contact between an environmental feature and

\footnotetext{
$\dagger$ Osuka and Kitajimas effort and ours are independent. We became aware of their work through their presentation/publication in October 2003. However, the development of our two serpentine robots, OmniPede and OmniTread, began in 1998 and September 2002, respectively.
}

a propulsion surface produces motion. We define the term "Propulsion Ratio," $P_{r}$, measured as the surface area that provides propulsion, $A_{p}$, divided by the total surface area of the body, $A_{p}+A_{i}$, where $A_{i}$ is the inert (non-propelling) surface area of the body. Our design goal is to maximize $P_{r}$.

Commonsense, supported by our experience, suggests that serpentine robots with a low propulsion ratio get stuck very easily when trying to move over rugged terrain. In order to increase the propulsion area, $A_{p}$, and thus the propulsion ratio, $P_{r}$, we cover all sides of the OmniTread with extra-wide tracks. We also took extensive measures to reduce the space (and thus, the inert area, $A_{i}$ ) between the segments, as will be explained in the next section.

\section{B. Joint actuation}

During our work with serpentine robots, we spent a significant amount of time on the analysis and formulation of requirements for joint actuators in serpentine robots. Listed here are the four most important ones:

1. By definition, serpentine robots are relatively long compared to their diameter, so that their lead segments can reach up and over a high obstacle while still being able to fit through small openings. However, lifting the lead segments requires a significant amount of torque, which is particularly difficult to generate in slender serpentine robots, where the lever arm for a longitudinal lifting force is limited by the robot's small diameter. One key requirement for serpentine robots is thus that they employ joint actuators of sufficient strength to lift up two or more of its lead or tail segments.

2. Another key requirement is that serpentine robots should conform to the terrain compliantly. This assures that as many driving segments as possible are in contact with the ground at all times, thereby providing effective propulsion. Serpentine robots that don't conform compliantly require extremely complex sensor systems to measure contact forces and to command a momentary angle for each non-compliant joint so as to force contact with the ground.

3. At times it is necessary to increase the stiffness of a joint for example to reach over an obstacle or for crossing a gap. Alternatively, it may be necessary to adjust the stiffness to an intermediate level, for example, when the lead segment leans against a vertical wall while being pushed up that wall by the following segments. Thus, serpentine robots must be capable of adjusting the stiffness of every DOF individually and proportionally.

4. Large amounts of space dedicated to joints increase the amount of inert surface area. Therefore, joint actuators should take up as little space as possible, to reduce the size of space occupied by joints (called "Joint Space").

Extensive studies of these requirements and of joint actuators potentially meeting these requirements led to the second unique design feature of the OmniTread: the use of pneumatic bellows for actuating the joints. Our research [Granosik and Borenstein, 2005] shows that pneumatic bellows meet all four of the above requirements better than any other type of actuator. In particular, pneumatic bellows provide a tremendous force-to-weight ratio, and they fit perfectly into the otherwise unusable (since varying) space between segments. The latter point is illustrated in Figure 2, 
which shows that parts of Joint Space may be small at one moment, and large at the next, depending on the bending of the joint. If we wanted to use Joint Space for housing electronics or other rigid components, then the size of that component would be limited by the dimensions of the "minimal space" shown in Figure 2. Contrary to rigid components, pneumatic bellows fit into such varying spaces perfectly: bellows expand and contract as part of their intended function, and they happen to be smallest when the available space is minimal and largest when the available space is maximal. From the point of space utilization, pneumatic bellows are thus a superbly elegant solution,

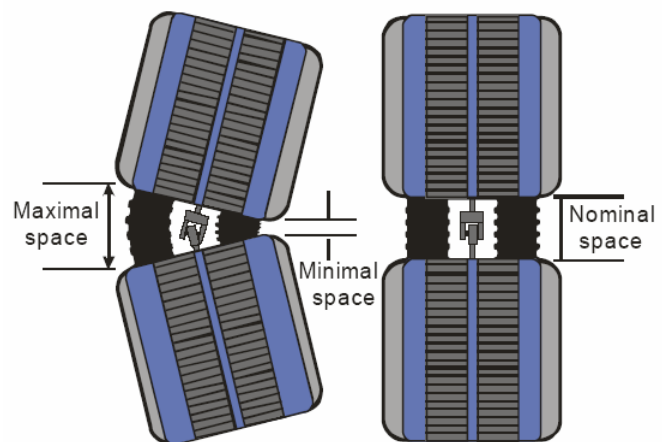

Figure 2: In serpentine robots the shape of so-called "joint space" varies with the angles of the joint. At extreme angles, in some regions of joint space there is almost no free space for mounting rigid components.

because joint actuators take up only Joint Space, and very little of it, for that matter. Therefore, we call our bellowsbased pneumatic system "Integrated Joint Actuator" (IJA). In contrast, pneumatic cylinders or McKibben muscles, as well as electric or hydraulic actuators, would all require space within the volume of the segments or much larger Joint Space. To further illustrate our point about small versus large Joint Spaces, we included Figure 3, which shows how the OmniTread successfully traverses a narrowedge obstacle, thanks to its very short joints. If the joints were longer than the rail's width, then the robot would necessarily get stuck on it.

\section{CONTROL OF THE INTEGRATED JOINT ACTUATOR}

In this section we present a solution for Requirement \#3 of Section II.B. This requirement, which calls for the proportional control of position and stiffness of the joints, is

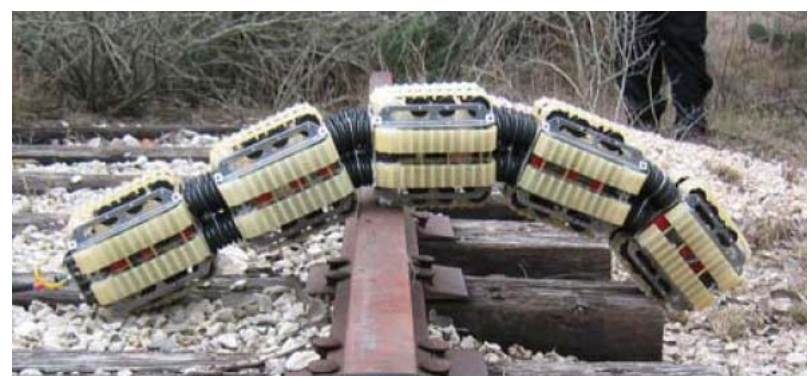

Figure 3: In the OmniTread, Joint Space is only $6.8 \mathrm{~cm}$ long while segments are $20 \mathrm{~cm}$ long. This design helps produce a very favorable Propulsion Ratio $P r$. The obvious advantage is the OmniTread's ability to cross a narrow-edged obstacle, such as this railroad rail. Robots with long inert Joint Spaces will get stuck on this narrow obstacle. not easy to meet with pneumatic actuators.

Traditionally, pneumatic systems were designed for socalled pick and place operations. Such designs are obviously too limited for the actuation of joints in serpentine robots. In the 90s, more advanced pneumatic control systems for proportional position control were introduced in the scientific literature (see [Granosik and Borenstein, 2004] for a review with our comments on these systems). None of the described systems, however, offers simultaneous control of position and stiffness, as is required for serpentine robots.

In order to provide both proportional position control and proportional stiffness control simultaneously, as well as zero-airflow at steady state, we developed what we call the "Proportional Position and Stiffness (PPS)" controller. Figure 4 shows a simplified 1-DOF joint operated by two bellows and controlled by the pneumatic circuit shown in Figure 5. Figure 6 shows the control block diagram for the PPS controller.

The PPS control algorithm is based on the simplified dynamic model of a bellows-driven joint, given by

$$
I \ddot{q}=\tau_{p}-\tau_{s}-\tau
$$

where:

$I$ - polar moment of inertia of parts rotating around joint ' $\mathrm{O}$ ' in Figure 4,

$q$ - joint angle;

$\tau_{s}-$ torque around ' $O$ ' Figure 4: A simplified 1-DOF joint with caused by elasticity

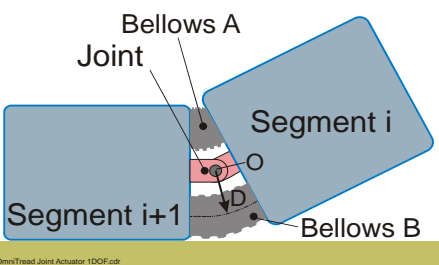

of inflated bellows. We observed that this component could be neglected for our bellows with no loss in the quality of trajectory following;

$\tau$ - load torque, generated by the weight of the lifted segment(s);

$\tau_{p}-$ net torque generated by Bellows $\mathrm{A}$ and $\mathrm{B}$, and given by

$$
\tau_{p}=D A\left(p_{A}-p_{B}\right)
$$

where:

$D$ - length of lever arm of torque-generating Bellows A and $\mathrm{B}$,

$A-$ cross section area of Bellows A and B.

$p_{A}, p_{B}-$ pressures in opposite Bellows A and B in Figure 4.

The actual control algorithm employed by our PPS controller is the so-called "inverse model" [Craig 1989] and is therefore shown as block "INV" in Figure 6. The task of Block INV is to generate reference values for the pressures $p_{r A}$ and $p_{r B}$, which are then passed on to the pressure controller. Block "INV" incorporates Eq. (1), which represents the sum of all torques acting in the simplified 1DOF joint of Figure 44. In steady state, the difference between the pressures in Bellows A and B determines the actuation moment that the bellows apply to the joint, as shown by Eq. (2). The sum of the pressures in Bellows A and $B$, determines the angular stiffness of the joint [Granosik and Jezierski 1999]. Thus, both the actuation torque and the angular stiffness can be controlled 


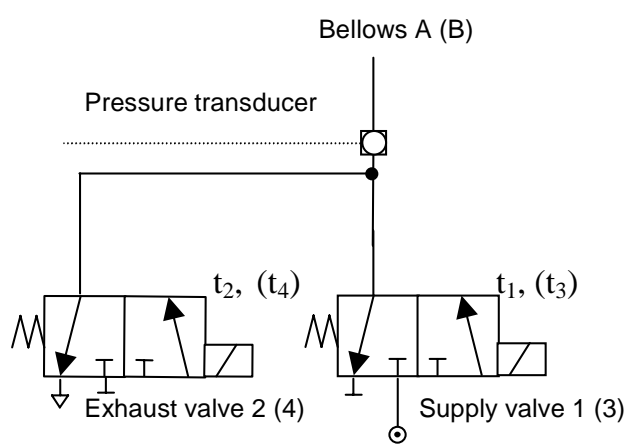

Figure 5: Pneumatic circuit for our Proportional Position and Stiffness (PPS) controller with zero air consumption at steady state. The location of Bellows A and B is shown in Figure 44.

simultaneously by selecting appropriate pressures $p_{A}$ and $p_{B}$.

The proposed control system assigns higher priority to stiffness when conflicts between position control and stiffness control arise. For example, conflicts may arise when the controller tries to reach a commanded position while being commanded to maintain low stiffness $S$. In this case, both $p_{A}$ and $p_{B}$ must be small, and the pressure difference, which produces the torque for moving the joint to the commanded position, may be to small to do so. In the case of such conflicts we assigned higher priority to stiffness control than to position control.

In order to derive our control approach, we make the important assumption that the dynamics of compressed air in a pair of bellows is similar to that in the two chambers of a cylinder. Based on this assumption, we adopted the expression derived by Shearer [1956] for the latter:

$$
\dot{p}=\frac{k R T \dot{m}}{V}-\frac{k p \dot{V}}{V}
$$

where:

$p, V, \dot{p}_{r}, \dot{V}$ - pressure in bellows, volume of bellows, and first derivatives of pressure and volume, respectively,

$k \quad-$ ratio of specific heats (for air $k=1.4$ ),

$R, \dot{m}, T$ - gas constant, mass airflow and temperature of compressed gas, respectively.

Pressures $p_{A}$ and $p_{B}$ are controlled in our system by means of pulse width modulation (PWM), which is realized in block $\mathrm{K}\left(p_{s}, p, q\right)$ in Figure 6. The PWM controller functions

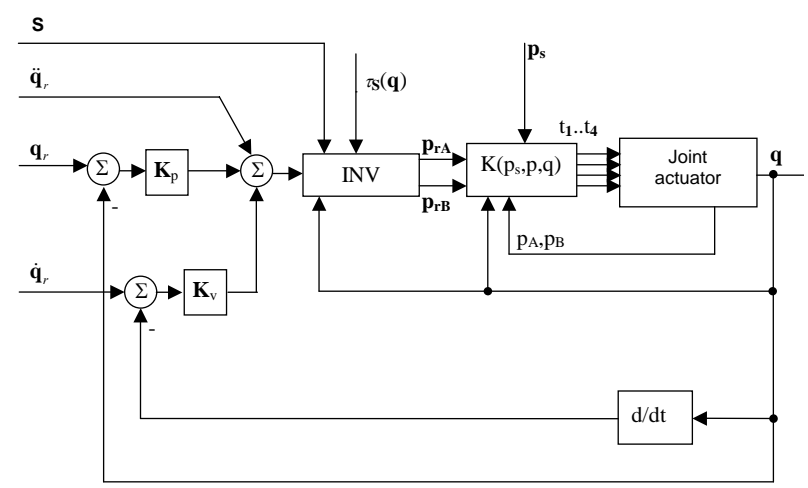

Figure 6: Block diagram of control system for our Proportional Position and Stiffness (PPS) controller with zero air consumption at steady state. by modifying the fraction of time $t_{i}(i=1 \ldots 4)$, during which certain valves of Figure 5 are open (in the case of exhaust valves) or closed (in the case of supply valves) during every PWM interval $t_{p}$.

We can compute the control parameter $t_{i}$ according to (4)

$$
t_{i}=\left\{\begin{array}{lll}
G \frac{t_{p}}{A_{s} \sqrt{p_{s}-p}} & \text { for } & G \geq 0 \\
G \frac{-t_{p}}{A_{e} p} & \text { for } & G<0
\end{array}\right.
$$

using energy flow $\mathrm{G}$ derived from (3) as:

$$
G=R k T \dot{m}=K_{D P} V \dot{e}_{p}+K_{P P} V e_{p}+k p \dot{V}
$$

where:

$t_{p}$-period of the pulse width modulation (PWM) controller,

$t_{i}$ - length of a pulse in the PWM controller. Four different signals $t_{1} . . t_{4}$ control the respective valves as shown in Figure 5. $t_{i}$ is always a fraction of $t_{p}$,

$e_{p}, \dot{e}_{p} \quad-$ pressure regulation error and its first derivative, respectively,

$K_{D P}, K_{P P}$ - derivative and proportional coefficients of PD pressure regulator.

$A_{s}=0.094$ and $A_{e}=-0.099$ are experimentally determined coefficients that apply for the case of $G>0$ and $G<0$, respectively. $G$ is proportional to mass airflow and its sign describes the direction of airflow. A positive value for $G$ indicates that air is being supplied to the bellows and that valve number 1 or 3 (see Figure 5) is closed. A negative value for $G$ indicates that air is being exhausted and that valves 2 or 4 are opened.

Figure 77 shows an experiment, in which a pair of pneumatic bellows was controlled to expand and contract in a sinusoidal fashion, for two full periods. The PPS controller maintains a near-constant stiffness of $20 \%$, as was commanded in this experiment. In our controller, stiffness does not have to be constant; it can change in the full range of $0 \%-100 \%$ under computer control and in real time. Both degrees of freedom of each joint are controlled independently using our PPS controller. However, to avoid possible conflicts we command the same stiffness for the entire joint.
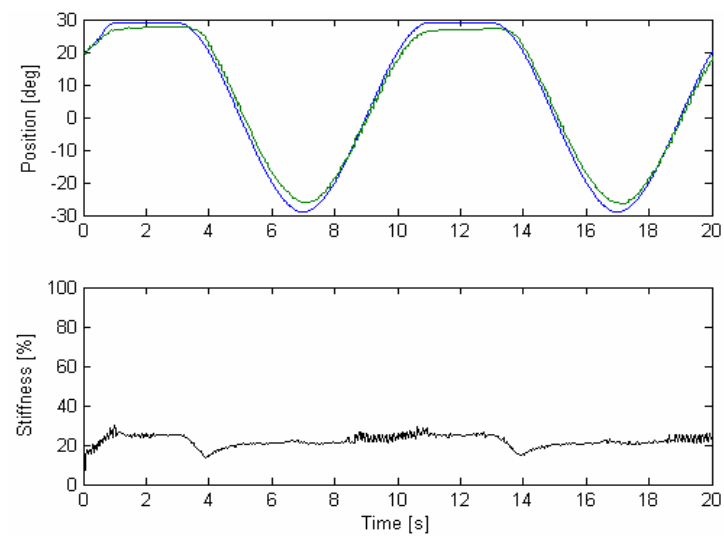

Figure 7: Experimental results with the PPS controller. Trajectory following with constant stiffness. 


\section{OMNITREAD - PERFORMANCE}

The OmniTread has five segments and four pneumatically actuated 2-degree-of-freedom (DOF) joints. The size of each segment is $20 \times 18.6 \times 18.6 \mathrm{~cm}$ (length $\times$ width $\times$ height). Each Joint Space is $6.8 \mathrm{~cm}$ long. The entire robot is $127 \mathrm{~cm}$ long and weighs about $13.6 \mathrm{~kg}$.

A single drive motor located in the center segment provides torque to all tracks on all five segments via a drive shaft running through the center of the segments. The four joints are actuated by a total of 16 pneumatic bellows. With this unique design the OmniTread has 33 individually and proportionally controllable parameters.

In order to control the OmniTread we developed a microprocessor-based distributed control system consisting of five local controllers - one for each of the OmniTread's four joints and a fifth controller for the electric drive motor. Each local controller is based on a 16-bit Motorola microcontroller MC9S12DP256B and all five controllers communicate with a master PC via CAN bus. Each local controller can receive new position and stiffness commands and return feedback data (two positions and four pressures) every $10 \mathrm{~ms}$. Upon receiving a command, the local controllers for the joints then sends pulse width modulation (PWM) signals to eight on-off pneumatic valves (two for each bellows and there are four bellows per 2-DOF joint).

In order to test the performance of the OmniTread under realistic and objective conditions, we tested the robot at the Small Robotic Vehicle Test Bed at the Southwest Research Institute (SwRI) in San Antonio, Texas.

During these tests the OmniTread was continuously controlled by two operators who had audio and visual contact with the robot, allowing them to monitor the robot's behavior at all times. Among the tests were tasks such as climbing over high steps, ascending through the inside of pipes, traversing wide gaps, any many more. Some numeric test results are summarized in Table I. Other performance results are best conveyed through the photographs in this section.

The feature of tracks-all-around the body of the OmniTread was quickly validated in some of the first tests. This is documented in Figure 8, where the robot traversed a natural (not man-made/urban) environment. Tracks on the sides of the robot provided additional propulsion, the lack of which might have stalled a vehicle without them. There were also abundant situations in which the robot rolled over sideways. Because of its almost complete coverage with propelling tracks, the robot continued operation without any difficulty.

Another unique OmniTread feature that fared well during the tests is the large amount of torque generated by

Table I: Performance specification of the OmniTread

\begin{tabular}{|l|l|}
\hline Maximum velocity & $10 \mathrm{~cm} / \mathrm{s}$ \\
\hline Minimum turning radius & $53 \mathrm{~cm}$ \\
\hline Maximum sideways slope & Concrete Ramp: $25^{\circ}$ \\
\hline Maximum incline & $\begin{array}{l}\text { Concrete Ramp: } 30^{\circ} \\
\text { Sand Ramp: } 15^{\circ}\end{array}$ \\
\hline $\begin{array}{l}\text { Maximum angle of slope of } \\
\text { PVC pipe (30 cm diameter) }\end{array}$ & $22^{\circ}$ \\
\hline Maximum height of curb & $45.7 \mathrm{~cm}$ \\
\hline Maximum gap traversed & $66 \mathrm{~cm}$ \\
\hline
\end{tabular}

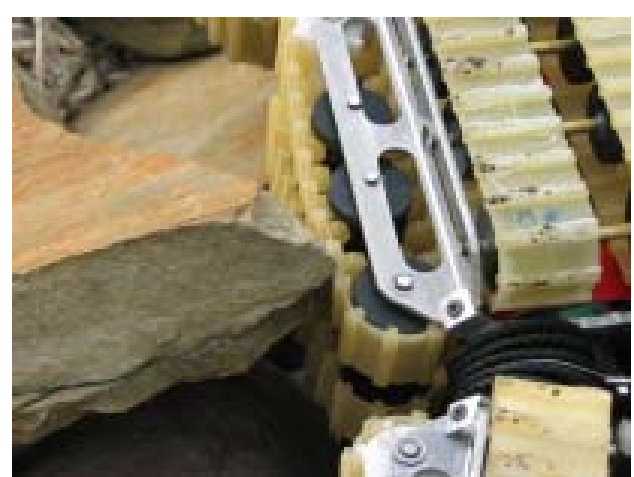

Figure 8: During a traverse of the "large rocks" site, we observed several episodes of rocks pressing into the OmniTread's side. Because of the "tracks-all-around" feature, the rocks didn't impede motion. Rather, they added propulsion.

the pneumatic bellows. This enables the robot to lift its two front (or rear) segments up, to reach up and over high steps. One impressive result obtained thanks to this feature was the successful climb up a $45.7 \mathrm{~cm}$ high curb, as shown in Figure 9a. This is about 2.3 times the height of the OmniTread. Such capabilities cannot be achieved by conventional tracked or wheeled mobile robots unless they are specially designed for the task.

Figure 9b shows the OmniTread entering a PVC pipe with an inside diameter of $30 \mathrm{~cm}$ and an inclination of $22^{\circ}$. Thanks to the OmniTread's powerful joint actuators, the robot is able to wedge itself between the upper and lower inside surfaces of the pipe and produce enough normal force against the inside walls of the pipe in order to climb up. Even though we do not explicitly control contact forces between OmniTread and the walls of the pipe, there is no danger of damaging the actuators due to their natural compliancy.

Figure 9c shows a sequence of photographs of the OmniTread traversing a $66 \mathrm{~cm}$ wide trench. This is actually just over $50 \%$ of the robots length. This seemingly impossible task can be accomplished by lifting the front segment up, which moves the center of gravity slightly toward the rear. A combination of high lifting power and high stiffness is needed to perform this manoeuvre.

\section{CONCLUSIONS}

This paper introduced a new mobile robot that belongs to the class of serpentine robots. Serpentine robots have the potential to provide hitherto unattainable capabilities, such as climbing over high steps, travel inside or outside of horizontal or even vertically pipes, or traversing wide gaps. While individual tasks of this nature have been tackled in the past by special-purpose mobile robots (e.g., pipe crawlers), it appears that only serpentine robots may be able to perform a large variety of difficult tasks. We believe that the OmniTread serpentine robot introduced here has a particularly high potential to become truly feasible and practical.

We are currently designing and building the next generation of the OmniTread, which we refer to as "OT-4." The OT-4 has half the diameter of the prototype described in this paper, and it will have seven segments (compared to the five segments of our present prototype). OT-4 will also 
have onboard power resources (electric and pneumatic) for over one hour of untethered operation.

\section{ACKNOWLEDGMENT}

This work was funded by the U.S. Department of Energy under Award No. DE-FG04-86NE3796 and by the Intelligence Technology Innovation Center (ITIC) through Southwest Research Institute under contract No. F009822.

We are also grateful to graduate student Malik Hansen for his work on designing and building the OmniTread segments.

\section{REFERENCES}

[1] BROWN, H.B., Jr.; Vande Weghe, J.M.; Bererton, C.A.; Khosla, P.K.; Millibot trains for enhanced mobility, IEEE/ASME Transactions on Mechatronics, Vol. 7, Issue 4, pp. 452-461, Dec. 2002

[2] CRAIG J.J., Introduction to robotics, Addison-Wesley Publishing Company, Inc. 1989

[3] GRANOSIK, G.; Borenstein, J.; Minimizing Air Consumption of Pneumatic Actuators in Mobile Robots, Proc. of the 2004 IEEE Int'l Conference on Robotics and Automation, pp. 3634-3639, New Orleans, LA, 2004

[4] GRANOSIK, G.; Borenstein, J.; Integrated Joint Actuator for Serpentine Robots, Submitted to the IEEE Transactions on Mechatronics. (2005)

[5] Granosik, G., Hansen, M., and Borenstein, J.; The OmniTread Serpentine Robot for Industrial Inspection and Surveillance. To appear in International Journal on Industrial Robots, Special Issue on Mobile Robots, vol. IR32-2, March 18th, 2005.

[6] GRANOSIK G., Jezierski E., Application of a maximum stiffness rule for pneumatically driven legs of walking robot, Proc. of $2^{\text {nd }}$ Int. Conference on Climbing and Walking Robots, pp.213-218, Portsmouth, UK 1999.

[7] HIROSE, S. and Morishima, A.; Design and Control of a Mobile Robot With an Articulated Body. The International Journal of Robotics Research, Vol. 9, No. 2, pp. 99-113, April 1990

[8] HIROSE, S., Morishima, A., Tukagosi S., Tsumaki T., Monobe H., Design of Practical Snake Vehicle: Articulated Body Mobile Robot KR-II, Fifth International Conference on Advanced Robotics, 'Robots in Unstructured Environments, pp 833 -838 vol.1, June 1991

[9] KIMURA, H.; Hirose, S.; Development of Genbu : Active wheel passive joint articulated mobile robot. Proc. IEEE/RSJ International Conference on Intelligent Robots and System, pp. 823 -828 vol.1, Oct. 2002

[10] KLAASSEN, B. and Paap, K.L.; GMD-SNAKE2: A Snake-Like Robot Driven by Wheels and a Method for Motion Control. Proc. of IEEE International Conference on Robotics and Automation. pp. 3014-3019, Detroit, MI, May 10-15,1999.

[11] OSUKA K, and Kitajima, H.; Development of Mobile Inspection Robot for Rescue Activities: MOIRA. Proceedings of the 2003 IEEE/RSJ Intl. Conference on Intelligent Robots and Systems, Las Vegas, Nevada • October 2003.

[12] SCHOLL, K.-U.; Kepplin, V.; Berns, K.; Dillmann, R.; Controlling a multi-joint robot for autonomous sewer inspection, Proc. IEEE International Conference on Robotics and Automation, ICRA '00., pp. 1701 -1706 vol.2, April 2000

[13] SHEARER J.L., Study of pneumatic processes in the continuous control of motion with compressed air I, II, Trans. ASME, pp. 233249, Feb.1956

[14] STREICH H., Adria O., Software approach for the autonomous inspection robot MAKRO, Proc. of the 2004 IEEE Int'l Conference on Robotics and Automation, pp. 3411-3416, New Orleans, LA, 2004.

[15] TAKAYAMA, T.; Hirose, S.; Development of Souryu-I connected crawler vehicle for inspection of narrow and winding space, 26th Annual Conference of the IEEE Industrial Electronics Society, IECON 2000., Page(s): 143 -148 vol.1, Oct. 2000.

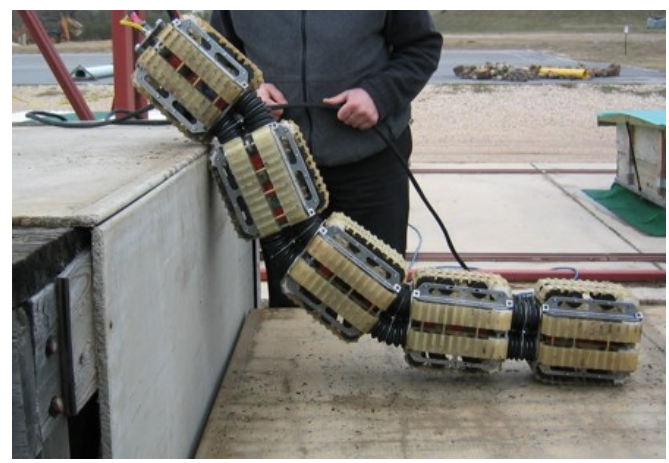

(a) OmniTread climbing up a 46-cm (18”) high step.

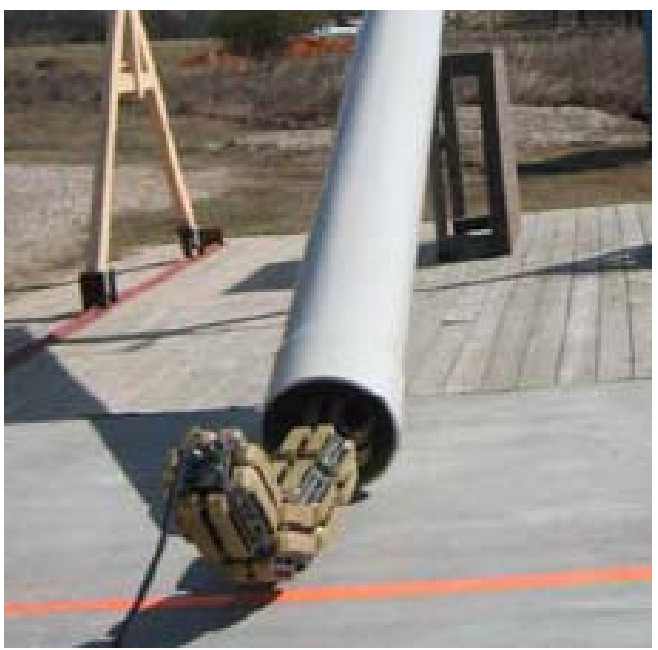

(b) OmniTread entering and climbing up inside a PVC pipe sloped at $22^{\circ}$.

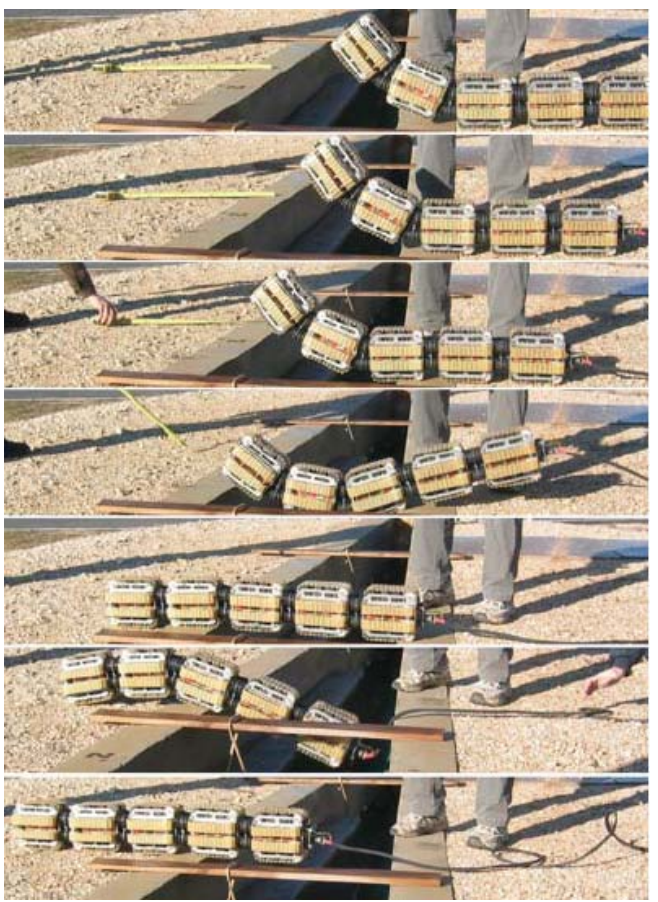

(c) OmniTread crossing a $66 \mathrm{~cm}$ (51\% of robot's length) wide trench

Figure 9: Situations during experiments in different test environments of the Small Robots test facility at the Southwest Research Institute (SwRI). 